\title{
Acute tryptophan depletion attenuates brain-heart coupling following external feedback
}

\author{
Erik M. Mueller ${ }^{1 *}$, Elisabeth A. Evers ${ }^{2}$, Jan Wacker ${ }^{1}$ and Freddy van der Veen ${ }^{3}$ \\ 'Department of Psychology, Philipps-Universität Marburg, Marburg, Germany \\ ${ }^{2}$ Department of Neuropsychology and Psychopharmacology, Maastricht University, Maastricht, Netherlands \\ ${ }^{3}$ Institute of Psychology, Erasmus University Rotterdam, Rotterdam, Netherlands
}

Edited by:

Tilmann A. Klein, Max Planck Institute for Human Cognitive and Brain Sciences, Germany

\section{Reviewed by:}

Redmond O'Connell, Trinity College Dublin, Ireland

Sören Enge, Technische Universität Dresden, Germany

\section{*Correspondence:}

Erik M. Mueller, Department of Psychology, Philpps-Universität Marburg, Gutenbergstrasse 18, 35032 Marburg, Germany. e-mail: erik.mueller@staff.unimarburg.de
External and internal performance feedback triggers neural and visceral modulations such as reactions in the medial prefrontal cortex and insulae or changes of heart period (HP). The functional coupling of neural and cardiac responses following feedback (cortico-cardiac connectivity) is not well understood. While linear time-lagged within-subjects correlations of single-trial EEG and HP (cardio-electroencephalographic covariance tracing, CECT) indicate a robust negative coupling of EEG magnitude $300 \mathrm{~ms}$ after presentation of an external feedback stimulus with subsequent alterations of heart period (the so-called $\mathrm{N} 300 \mathrm{H}$ phenomenon), the neurotransmitter systems underlying feedback-evoked cortico-cardiac connectivity are largely unknown. Because it has been shown that acute tryptophan depletion (ATD), attenuating brain serotonin $(5-\mathrm{HT})$, decreases cardiac but not neural correlates of feedback processing, we hypothesized that 5-HT may be involved in feedback-evoked cortico-cardiac connectivity. In a placebo-controlled double-blind cross-over design, 12 healthy male participants received a tryptophan-free amino-acid drink at one session (TRP-) and a balanced amino-acid control-drink (TRP+) on another and twice performed a time-estimation task with feedback presented after each trial. $\mathrm{N} 300 \mathrm{H}$ magnitude and plasma tryptophan levels were assessed. Results indicated a robust $\mathrm{N} 300 \mathrm{H}$ after $\mathrm{TRP}+$, which was significantly attenuated following TRP-. Moreover, plasma tryptophan levels during TRP+ were correlated with $\mathrm{N} 300 \mathrm{H}$ amplitude such that individuals with lower tryptophan levels showed reduced $\mathrm{N} 300 \mathrm{H}$. Together, these findings indicate that 5-HT is important for feedback-induced covariation of cortical and cardiac activity. Because individual differences in anxiety have previously been linked to 5-HT, cortico-cardiac coupling and feedback processing, the present findings may be particularly relevant for futures studies on the relationship between 5-HT and anxiety.

Keywords: feedback processing, FRN, tryptophan depletion, serotonin, vagal, heart rate

\section{INTRODUCTION}

We rapidly process internal and external feedback signals in order to optimally interact with our environment. Neural signatures of error or negative feedback processing (e.g., event-related potentials, fMRI activation) are often accompanied by changes of behavior (Debener et al., 2005; Eichele et al., 2010; Mueller et al., 2011): the commission of an error in a speeded reaction time task can lead to subsequent slowing of reaction times; the presentation of negative feedback following a particular decision can alter decision-making in the future. The adaptiveness of such behavioral changes with regard to task performance is still under investigation (Notebaert et al., 2009). Negative feedback in real life (e.g., a loose stone when climbing a rock) may often indicate a sudden need for intensive and effortful compensatory actions (quickly grasping another stone), associated with a sudden requirement for energy. Consistently, it has also been demonstrated that error and feedback signals trigger reactions in the periphery such as changes in heart rate (Crone et al., 2003; Hajcak et al., 2003; Wessel et al., 2011). Because these peripheral reactions are sensitive to feedback valence, even in the case of abstract feedback stimuli, which can hardly be adequately represented at the subcortical level, it is likely that cortical structures are involved in the modulation of cardiac responses at some point.

Consistent with this proposal Mueller et al. (2010) recently demonstrated a robust covariation between cortical (e.g., EEG) and subsequent cardiac chronotropic (i.e., heart period) modulations. Using a probabilistic gambling task (Sato et al., 2005), it was shown that a feedback stimulus indicating whether participants won or lost a small amount of money elicited among others (a) a large frontomedial amplitude which peaked at $300-400 \mathrm{~ms}$ and (b) a relative acceleration of heart rate which occurred around 3-4 s later. Most importantly, using a novel within-subject singletrial correlation method termed Cardio-Electroencephalographic Covariance Tracing (CECT) it was shown, that regardless of whether a win or loss was signaled, the frontomedial single-trial EEG-amplitude at $300-400 \mathrm{~ms}$ correlated with subsequent cardiac acceleration in the same trial. That is, a relatively larger positivity $300 \mathrm{~ms}$ after feedback presentation predicted relatively 
more cardiac acceleration $4 \mathrm{~s}$ later. Because this phenomenon is reflected in a negative within-subject correlation between EEG amplitude at $300 \mathrm{~ms}$ and subsequent heart interbeat interval (IBI) changes we termed it $\mathrm{N} 300 \mathrm{H}$. The $\mathrm{N} 300 \mathrm{H}$ was robust across individuals in the initial study (Mueller et al., 2010) and was recently replicated in another sample (Panitz et al., 2011). In both studies, the $\mathrm{N} 300 \mathrm{H}$ did not differ between positive and negative feedback valence. Using a low resolution localizing approach Panitz et al. (2011) associated the $\mathrm{N} 300 \mathrm{H}$ with increased insula current source density $300 \mathrm{~ms}$ after feedback presentation-an observation converging with the importance of the insular cortices for autonomic regulation (Gianaros et al., 2004).

The finding that feedback-locked EEG with a latency of $300 \mathrm{~ms}$ relates to heart period modulations converges with prior work on the P300 event-related potential component. It has long been speculated that P300 and evoked changes in autonomic activity are linked to each other, for example as different correlates of the orienting response which may function to facilitate information processing and/or action preparation (Graham and Clifton, 1966; Donchin, 1981; Donchin et al., 1984; Rushby et al., 2005; Polich, 2007; Nieuwenhuis et al., 2011). Both, P300 and evoked changes of heart period are modulated by motivational significance (Duncan-Johnson and Donchin, 1977; Fowles et al., 1982; Sato et al., 2005), which could vary across feedback presentations and thereby induce cortico-cardiac covariation as indicated by $\mathrm{N} 300 \mathrm{H}$. Finally, because one putative generator of the P300 is the insula (Linden, 2005), there appears to be structural overlap between sources linked to P300 and to N300H.

The neurotransmitter systems involved in this feedbackrelated cortico-cardiac connectivity phenomenon are currently unknown. Given the plethora of brain structures involved in feedback processing and the neurogenic modulation of peripheral responses to external stimuli (Benarroch, 1997; Ullsperger and von Cramon, 2003; Critchley et al., 2005; Wager et al., 2009) several transmitter systems including dopaminergic, noradrenergic, and serotoninergic processes may be of relevance. While dopamine may play an important role for modulating cortical and behavioral action monitoring processes (Holroyd and Coles, 2002; Ullsperger, 2010; Mueller et al., 2011), in the present paper we focus on the serotonergic system, which may be of particular relevance for transmitting cortically processed feedback information to the viscera.

In vitro studies have shown that several 5-HT receptor subtypes are expressed at vagal nuclei binding sites and at the nucleus tractus solitarius (Manaker and Verderame, 1990; Thor et al., 1992), which modulates heart rate indirectly (e.g., by its projections to the nucleus ambiguous). Moreover, it has been demonstrated in a variety of animal studies, that central 5 -HT is involved in control of cardiac vagal output (Jordan, 2005). Because feedback-related alterations in heart period show a relatively short latency (e.g., 1-3 s; Crone et al., 2003; 3$4 \mathrm{~s}$ : Mueller et al., 2010), it can be assumed that quick vagal rather than slow sympathetic modulations (Berntson et al., 1997) underlie feedback-evoked cardiac responses. Therefore, by affecting cardiac vagal output, 5-HT may modulate the transmission of feedback-related information from the brain to the viscera.
In line with this assumption, van der Veen et al. (2008) demonstrated, that a manipulation of the 5-HT system modulated peripheral (i.e., heart rate) but not central (i.e., EEG) responses to a negative feedback stimulus. Specifically, participants were tested at two sessions. In a double-blind crossover design participants received either a drink that did or a drink that did not contain the important 5-HT precursor tryptophan, thereby presumably increasing or lowering central 5-HT synthesis and release (Bell et al., 2001). At both sessions, participants performed a time-estimation task (Miltner et al., 1997), in which performance feedback was given after each trial. The authors analyzed the feedback-related negativity as a cortical indicator for feedback processing and heart IBI for the subsequent heart beats as a cardiac marker for feedback reactivity. Following the drink that did contain tryptophan (TRP+), it was shown, that feedback induced an increase in FRN amplitude for negative vs. positive feedback and a (valence independent) decrease of heart period for the three following heartbeats. Following the drink that did not contain tryptophan $(\mathrm{TRP}-)$, negative feedback induced an increase in FRN amplitude that was comparable to the TRP+ session. However, the relative cardiac deceleration after negative feedback was significantly smaller after TRP - as compared to TRP+.

One explanation of these findings is, that a cortical feedbackrelated process, which is not captured by the FRN but associated with the cardiac response is attenuated by $5-\mathrm{HT}$. In addition, the findings by van der Veen et al. (2008) could indicate, that 5-HT influences how cortically processed feedback-information is subsequently transmitted to the periphery. Under the assumption, that the $\mathrm{N} 300 \mathrm{H}$ captures such cortico-cardiac transmission following feedback presentation (Mueller et al., 2010), it could thus be hypothesized that TRP-, by lowering central 5-HT, reduces feedback-related $\mathrm{N} 300 \mathrm{H}$ values compared to TRP+. This hypothesis was tested in the present study by reanalyzing the data of van der Veen et al. with the CECT-approach.

\section{METHODS \\ PARTICIPANTS}

Data of 12 healthy male participants (mean age: 22.5 years; $S D=5$ years) with complete EEG and ECG data for both sessions (TRP+ and TRP-) was available from the study previously published by van der Veen et al. (2008).

\section{STIMULI}

Participants performed a time estimation task based on a paradigm developed by Miltner et al. (1997) and the details of the task can be found elsewhere (van der Veen et al., 2008). Participants had to estimate a $1 \mathrm{~s}$ interval starting at the onset of a visual cue by pushing a button and received feedback $1 \mathrm{~s}$ after the given response. An exclamation mark ("!”) was presented if the estimation was correct, a "+" was presented when the estimation was too long and a "-" was presented when the estimation was too short. The feedback stimulus was presented for $1 \mathrm{~s}$ and was followed by a $2 \mathrm{~s}$ inter-trial interval. Percentage of positive and negative feedback was kept at about $50 \%$ by varying the interval in which the estimation was labeled as correct. 


\section{PROCEDURE}

Participants were tested in a randomized double-blind crossover design and details are described elsewhere (van der Veen et al., 2008). Participants were tested on 2 separate days. On 1 day $(\mathrm{TRP}-)$ they ingested the tryptophan depleted mixture which contained $200 \mathrm{ml}$ of tap water plus $75 \mathrm{~g}$ of the amino-acids Lalanine, L-glycine, L-histidine, L-isoleucine, L-leucine, L-lysine, L-phenylalanine, L-proline, L-serine, L-threonine, L-tyrosine, Lvaline, L-arginine, L-cysteine, and L-methionine. On the other day (TRP+) they ingested the balanced amino-acid mixture which contained the same ingredients plus $3.0 \mathrm{~g}$ tryptophan. Participants were tested $5 \mathrm{~h}$ after ingestion of the mixture. Of each participant, a blood sample was taken right before drinking the amino acid mixture and right before the psychophysiological assessment, i.e., about $5 \mathrm{~h}$ after drinking the amino-acid mixture. As described in van der Veen et al. (2008), the raw plasma concentration of free tryptophan was determined from these samples.

\section{EEG AND ECG}

As described in detail elsewhere (van der Veen et al., 2008), ECG was derived from pre-cordial leads and was sampled at $1000 \mathrm{~Hz}$. $\mathrm{R}$ spikes were detected online with an accuracy of $1 \mathrm{~ms}$, manually checked and corrected if necessary. The ECG was converted into a (stepwise) IBI-time course with a sampling rate of $500 \mathrm{~Hz}$ where the value at each sample reflects the distance between the preceding and succeeding $r$-spike in $\mathrm{ms}$. EEG was recorded at sensors F3, Fz, F4, C3, Cz, C4, Pz, and at one ocular channel using a sampling rate of $500 \mathrm{~Hz}$. Epochs with non-systematic artifacts in either EEG or IBI were manually removed while Independent Component Analysis was used to correct for ocular artifacts in the EEG.

\section{CECTs}

To compute CECTs, the continuous EEG was first segmented into epochs from 0 to $500 \mathrm{~ms}$ relative to the feedback stimulus and baseline corrected for the preceding $500 \mathrm{~ms}$. EEG epochs were then binned into 50 bins of $10 \mathrm{~ms}$ each (corresponding to a sampling rate of $100 \mathrm{~Hz}$ ) and mean voltage magnitudes were determined for each bin. With this data, for each participant and each channel an EEG matrix for subsequent CECT analyses was constructed which consisted of about 200-240 rows (depending on the number of artifact-free trials) and 50 columns (corresponding to the 50 bins per epoch). In analogy to the EEG, the continuous IBI-trace was segmented into epochs from 0 to $2500 \mathrm{~ms}$ relative to the feedback stimulus and baseline corrected for the preceding $250 \mathrm{~ms}$. IBI epochs were binned into 10 bins of $250 \mathrm{~ms}$ each $(4 \mathrm{~Hz})$ and mean IBI values were determined for each bin. For each participant an IBI matrix for CECT analyses was constructed that consisted of about 200-240 rows (trials) and 10 columns (bins). CECT matrices were computed for each participant and channel, separately by correlating each column of the corresponding EEG matrix with each column of the IBI matrix. Thus, for every CECT matrix $10 \times 50$ within-subject correlations were computed (correlations over trials). These were now structured in a $10 \times 50$ correlation matrix in which each row represents one particular IBI bin and each column represents one particular EEG bin. To illustrate the interpretation of CECT matrices, a correlation displayed in column 1, row 2, reflects how much the EEG magnitude from 0 to $10 \mathrm{~ms}$ (EEG bin 1) after a feedback stimulus correlates with the change in IBI from 250 to $500 \mathrm{~ms}$ after a feedback stimulus (IBI bin 2).

Grand average CECTs (as displayed in Figure 1) were computed by averaging the CECT matrices across participants (separately for each channel). To first test for an effect of acute tryptophan depletion (ATD) a mean CECT-value (averaged across all time bins) was compared between TRP+ and TRP - session using a paired-samples $t$-test. To then test whether an $\mathrm{N} 300 \mathrm{H}$ component could be replicated in the present study, CECT-values were tested against zero (across participants, separately for each session) by conducting one-sample $t$-tests for each cell of the CECT-matrix at channel $\mathrm{Cz}(p<0.05$, twosided). With this analysis an $\mathrm{N} 300 \mathrm{H}$ like component was evident in the TRP+ session, which extended from 150 to $300 \mathrm{~ms}$ in the EEG time domain and from 250 to $1750 \mathrm{~ms}$ in the IBI time domain. To assess the between-subject correlation of $\mathrm{N} 300 \mathrm{H}$ and tryptophan level we extracted individual peak CECT-values in this time window (see Figure 1). To test for effects of feedback valence, separate CECTs were computed for positive and negative feedback and mean CECT-values in the previously specified timewindow were tested against zero and compared between feedback types.

\section{RESULTS}

\section{THE EFFECT OF ATD ON PLASMA TRYPTOPHAN LEVEL}

As expected and as previously reported for the entire sample, the oral intake of the tryptophan-deficient amino-acid mixture reduced the levels of free tryptophan concentrations in blood plasma from $M=53(\mathrm{SD}=6)$ at $T_{0}$ (i.e., before the drink was taken) to $M=17(\mathrm{SD}=8)$ at $T_{5}$ (i.e., $5 \mathrm{~h}$ after the drink was taken just before the task began) $\left(t_{(11)}=12.28, p<0.001\right)$. In contrast, the ingestion of the nutritionally balanced mixture at the TRP + session yielded an increase of plasma tryptophan levels from $T_{0}(M=50 ; \mathrm{SD}=14)$ to $T_{5}(M=105 ; \mathrm{SD}=41)$ $\left(t_{(11)}=5.99, p<0.001\right)$.

\section{BEHAVIORAL PERFORMANCE}

On average, participants had 112 correct responses, 40 underestimations and 88 over-estimations of the $1 \mathrm{~s}$ epoch (the window was continuously adapted to provide about equal amounts of positive and negative feedback). There was no effect of ATD on the number of correct responses, under-estimations or overestimations ( $p s>0.5)$.

\section{EVENT-RELATED POTENTIAL AND EVEN-RELATED CARDIAC RESPONSE}

Feedback-evoked a P300-like deflection, which extended from 200 to $500 \mathrm{~ms}$ (peak at $360 \mathrm{~ms}$, channel Cz) and a triphasic cardiac response (0-1000 ms: deceleration; 1000-2000 ms: acceleration; 2000-2500 ms: deceleration). ATD did not influence the P300 amplitude $(p>0.5)$, but reduced the cardiac response to feedback for bins 3, 4, and 5 (i.e., 500-1250 ms; $p<0.05$ ). Effects of feedback-valence were not analyzed in the present report (see van der Veen et al., 2008, for effects of feedback-valence). 


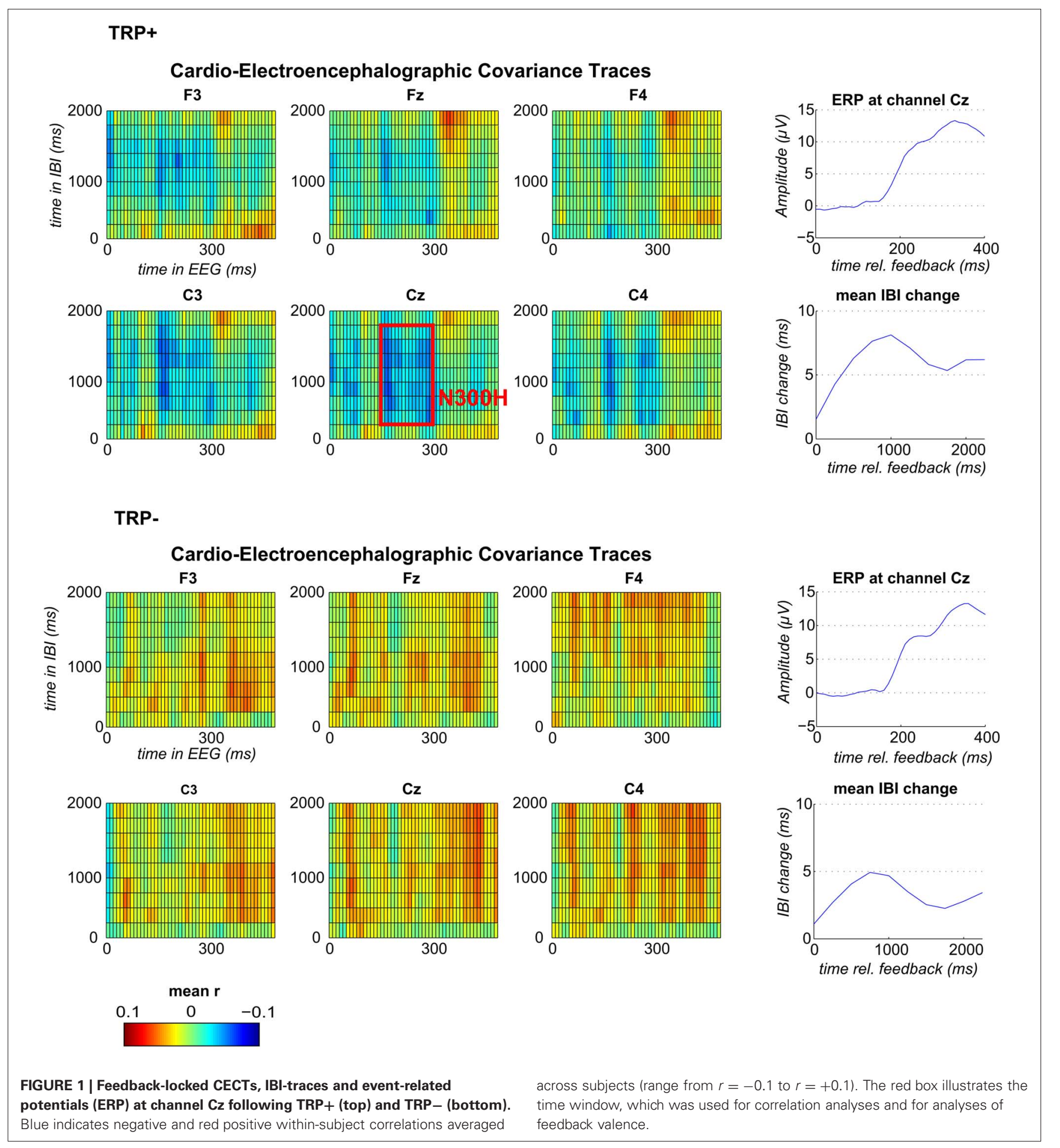

\section{CARDIO-ELECTROENCEPHALOGRAPHIC COVARIANCE TRACING Effects of session on the overall CECT}

A paired samples $t$-test comparing mean CECT-values (whole CECT epoch from 0 to $500 \mathrm{~ms}$ in the EEG and 0 to $2500 \mathrm{~ms}$ in the IBI time domain) between TRP + and TRP - session indicated a significant effect of session for the overall CECT $\left(t_{(11)}=3.26\right.$, $p<0.01)$.

\section{Replication of $\mathbf{N 3 0 0 H}$}

Because ATD affected the overall CECT, separate CECTs for each session were tested for $\mathrm{N} 300 \mathrm{H}$. As shown in Figures 1 and 2 CECT-analyses for the TRP+ session revealed an $\mathrm{N} 300 \mathrm{H}$-like component that was negative and significantly different from zero ( $p<0.05$, two-sided, 19 adjacent time-bins). The peak average within-subject correlation within this cluster was at $280 \mathrm{~ms}$ in the 


\section{Cardio-Electroencephalographic Covariance Traces (t-values; Cz)}
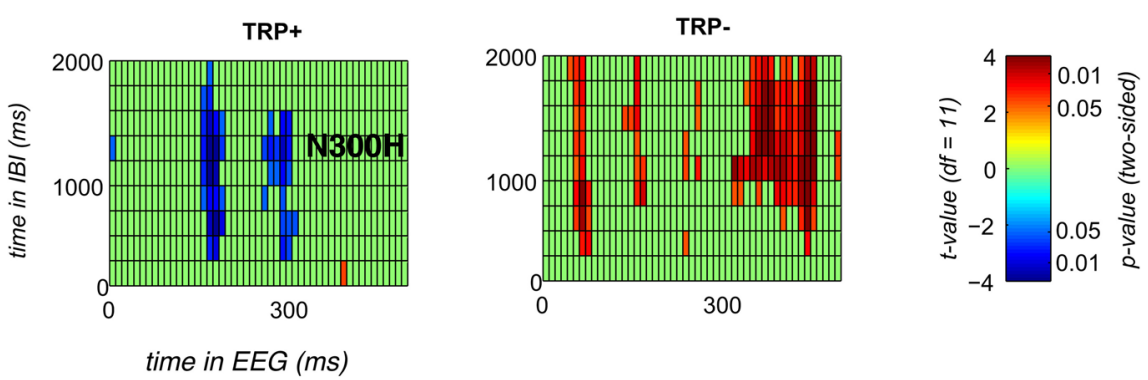

FIGURE 2 | Significant $\boldsymbol{t}$-values for feedback-locked CECTs at channel Cz following placebo (left) or acute tryptophan depletion (right). $T$-values are thresholded with $p<0.05$ (two sided). Any non-significant $t$-values are plotted in green color.

EEG and at 1250-1500 $\mathrm{ms}$ in the IBI time domain $\left(t_{(11)}=-3.6\right.$, $p<0.005)$. There was also a slightly earlier portion of the EEG (150-180 ms) that showed a significant negative covariation with subsequent IBI values. It should be noted that the small sample size (and consequently limited statistical power) precluded application of a conservative Bonferroni-correction for $10 \times 50$ tests (resulting in $\alpha^{\prime}=0.0001$ ), which might be considered appropriate in the light of the limited a priori knowledge about the timing of $\mathrm{N} 300 \mathrm{H}$ in the present task (Mueller et al., 2010). The statistical power of such Bonferroni-corrected $t$-tests is $<0.09$, which were (unsurprisingly) non-significant.

Of relevance, in the TRP - session an unexpected, temporally less specific positive association between feedback-evoked EEG and IBI values reached significance $(p s<0.05)$. As shown in Figures 1 and 2, a negative $\mathrm{N} 300 \mathrm{H}$ like component could not be observed following ATD (all ps > 0.05).

\section{Effects of feedback valence and ATD on $\mathrm{N3OOH}$}

Mean $\mathrm{N} 300 \mathrm{H}$ values in the time window from 150 to $300 \mathrm{~ms}$ in the EEG time domain and from 250 to $1750 \mathrm{~ms}$ in the EEG time domain were significantly different from zero for positive $\left(t_{(11)}=2.23, p<0.05\right)$ and negative $\left(t_{(11)}=2.50, p<0.031\right)$ feedback in the TRP + but not TRP - condition ( $p s>0.09$, twosided). A TRP $\times$ Feedback-Valence ANOVA yielded a main effect for TRP $\left(F_{(1,12)}=12.6, p<0.005\right)$, confirming the findings for the valence-independent CECTs reported above. There was also a trend for a TRP $\times$ Valence interaction $F_{(1,12)}=3.5, p<0.09$, indicating that $\mathrm{N} 300 \mathrm{H}$ to negative feedback tended to be less affected by ATD than $\mathrm{N} 300 \mathrm{H}$ to positive feedback. Consistent with Mueller et al. (2010), there was no main effect of feedback valence on $\mathrm{N} 300 \mathrm{H}$.

\section{Correlations between plasma tryptophan level and $\mathrm{N300H}$}

In addition to these group effects, we examined the association between plasma tryptophan level and $\mathrm{N} 300 \mathrm{H}$ aiming to probe whether interindividual variations of tryptophan level are also related to $\mathrm{N} 300 \mathrm{H}$. The tryptophan level was measured at two occasions $\left(T_{0}\right.$ and $\left.T_{5}\right)$ on each day $(\mathrm{TRP}+$ and TRP -$)$. Overall, the $T_{0}$ tryptophan level at the TRP + day was correlated with the $T_{5}$ tryptophan level at TRP + day $(r=0.74, p<0.005$, onesided) and $T_{0}$ tryptophan level at TRP- day $(r=0.54, p<0.05$, one-sided), indicating that interindividual differences in the tryptophan level were reliably measured and relatively stable over time, although the average tryptophan level was elevated by the nutritionally balanced drink as reported above. Importantly, the tryptophan level at TRP+ day (averaged across $T_{0}$ and $T_{5}$ ) was significantly correlated with $\mathrm{N} 300 \mathrm{H}$ at the TRP+ day $(r=-0.68$, $p<0.05$, Figure 3). Consistent with the experimental findings, the direction of these correlations indicates that a reduced level of plasma tryptophan predicted lower (i.e., less negative) $\mathrm{N} 300 \mathrm{H}$. As reported above at $T_{5}$ of the TRP- day, plasma tryptophan levels were severely reduced. These reduced tryptophan levels were not significantly associated with the (attenuated) $\mathrm{N} 300 \mathrm{H}$ in that session ( $p>0.4$, Figure 3), possibly due to blunted betweensubject variation after $\mathrm{TRP}-(\mathrm{SD}=8 \mu \mathrm{Mol} / \mathrm{l})$ vs. $\mathrm{TRP}+(\mathrm{SD}=$ $41 \mu \mathrm{Mol} / \mathrm{l})$. Together, these correlative findings indicate that baseline variations of plasma tryptophan levels are also related to $\mathrm{N} 300 \mathrm{H}$.

\section{CONTROL ANALYSIS}

To rule out the alternative explanation that ATD reduced corticocardiac within-subject correlations by affecting trial-to-trial

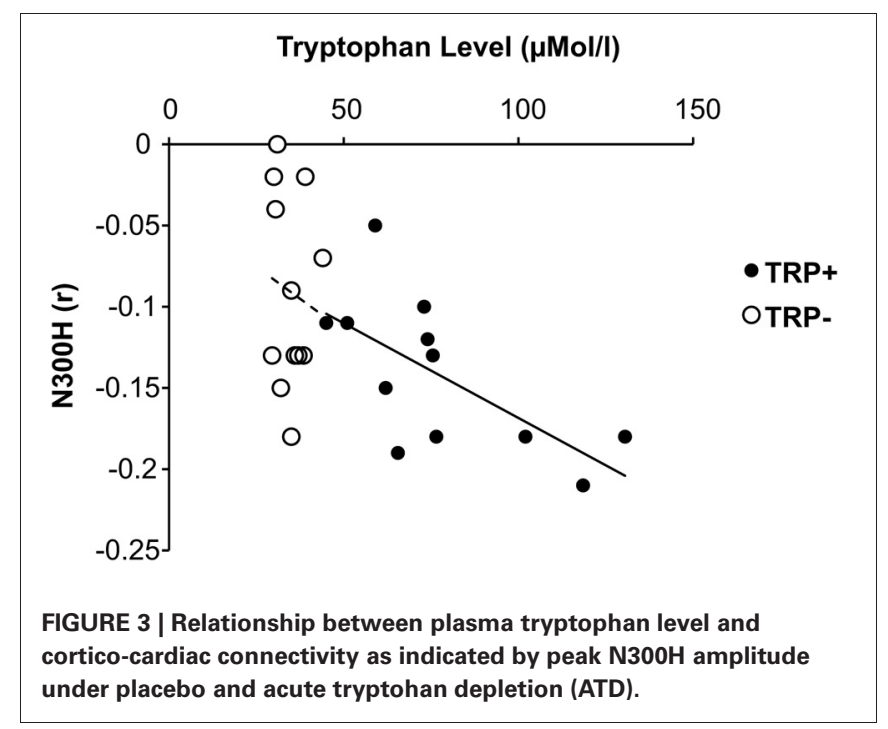


variation of cardiac responses we tested whether there was a difference in the cross-trial variance of feedback-locked IBI (from 250 to $1750 \mathrm{~ms}$ ) between TRP+ and TRP-. However, this was not the case $(p>0.5)$.

\section{DISCUSSION}

The goal of the present study was to test whether a manipulation of central 5-HT would influence cortico-cardiac connectivity evoked by feedback stimuli. Central 5-HT was manipulated through administration of drinks that either contained no tryptophan (TRP-), serving to deplete this precursor of 5-HT synthesis, or elevated tryptophan levels (TRP + ) in two separate sessions (double-blind cross-over design). Five hours later, when plasma levels of tryptophan were reduced in the TRP - vs. TRP + session, participants conducted a time estimation task in which feedback was given after each trial. To measure cortico-cardiac connectivity, CECT-analyses were conducted, in which feedbackevoked-changes in single-trial EEG magnitudes are systematically correlated with time-lagged feedback-evoked changes in heart period. Importantly, overall CECTs were significantly different between the TRP + and TRP - session. In the TRP + session, we replicated a previously reported phenomenon $(\mathrm{N} 300 \mathrm{H})$, indicating a significant coupling between centromedial EEG magnitude about $300 \mathrm{~ms}$ after feedback presentation and subsequent accelerations of heart rate (i.e., smaller IBIs). Of particular relevance, $\mathrm{N} 300 \mathrm{H}$ was absent following $\mathrm{TRP}-$, indicating that a pharmacological downregulation of 5-HT synthesis attenuated the negative covariation of feedback-evoked EEG and heart period changes or even induced a positive covariation (as indicated by the red spots in Figures 1 and 2). Moreover, interindividual differences in plasma tryptophan levels at the TRP+ session were correlated with $\mathrm{N} 300 \mathrm{H}$ values measured in the same session. Together these findings provide converging evidence that tryptophan plays a role in the covariation of cortical and cardiac feedback-related activity as indicated by $\mathrm{N} 300 \mathrm{H}$. One possible interpretation of these preliminary findings is that 5-HT is crucial for transmitting feedback-related information from the brain to the viscera.

As outlined in Figure 4 cortico-cardiac coupling following feedback presumably involves several structures, which may communicate in parallel and bidirectionally. The cortical processing of feedback includes the anterior (mid-) cingulate cortex and the insulae (Ullsperger and von Cramon, 2003), two highly relevant regions for central autonomic control (Benarroch, 1997; Gianaros et al., 2004; Critchley, 2005). Via projections to key regions in midbrain and pons these feedback-relevant structures can indirectly modulate input to the nucleus tractus solitarus in the dorsal medulla and the nucleus ambiguous [path (a) and (b)], where cell bodies of the myelinated cardiac vagal preganglionic neurons [path (c)] are located (Benarroch, 1997; Jordan, 2005). Accordingly, feedback-related information can be transmitted relatively quickly from the prefrontral cortex (where stimulus valence of rather abstract stimuli can be assessed) to the heart (where modulations may prepare subsequent alterations of behavior).

In the present study CECTs indicated a covariation of feedback-evoked single-trial EEG at $300 \mathrm{~ms}$ and changes in IBI from 250 to $1750 \mathrm{~ms}$ in the TRP+ session $(\mathrm{N} 300 \mathrm{H})$. Because the

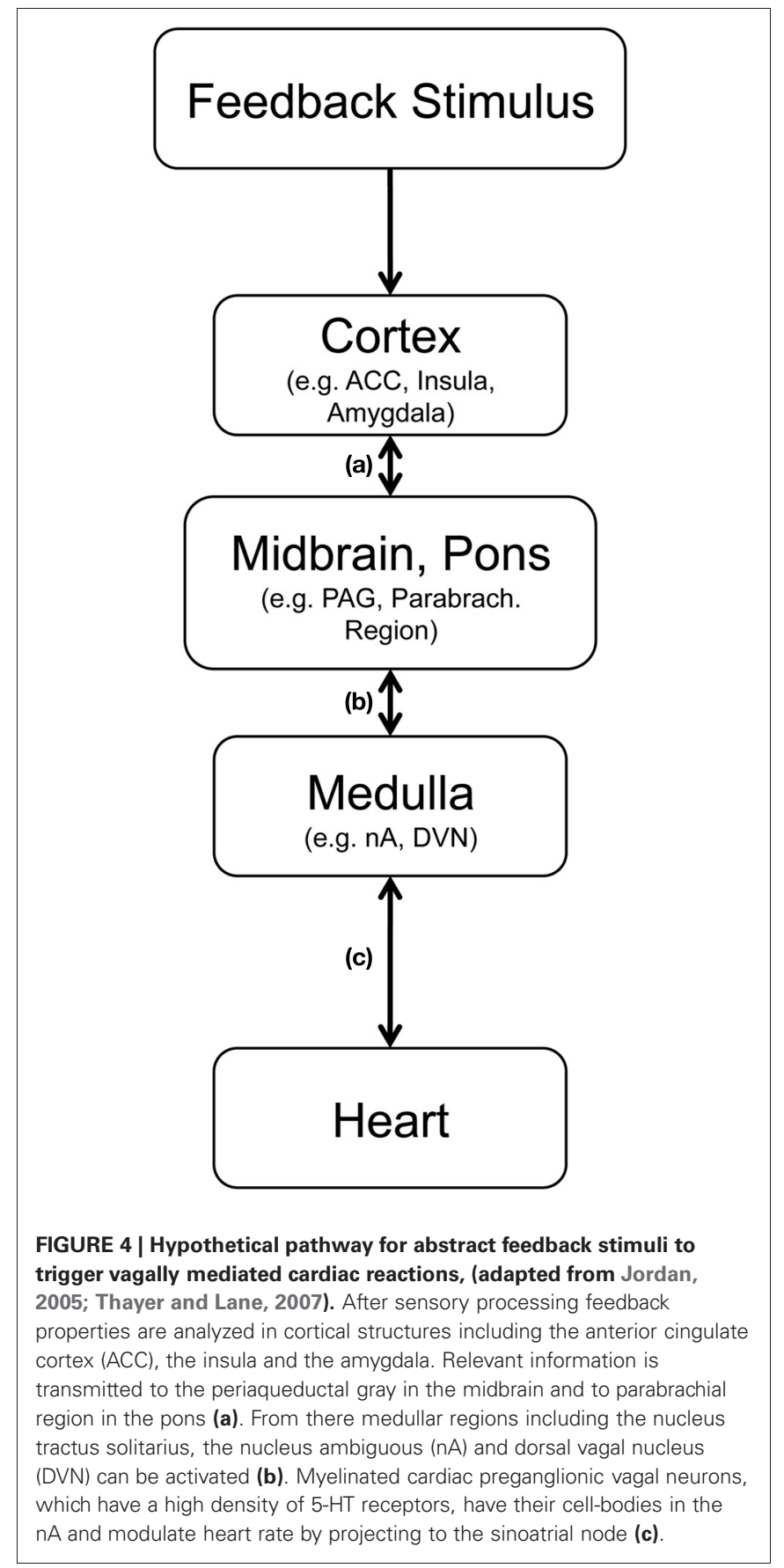

EEG in the TRP+ session thus correlated with an earlier proportion of IBI than in the Mueller et al. (2010) study (3000-4000 ms) and because a different paradigm was used in the two studies, it is possible that this cortico-cardiac covariation reflects a different phenomenon than $\mathrm{N} 300 \mathrm{H}$ in the Mueller et al. (2010) study. It could be hypothesized, that tasks with short intertrial intervals and/or tasks where feedback crucially depends on participants' performance (like in the current study) trigger earlier neurogenic accelerations of heart period (as observed in the event-related cardiac responses of the current vs. Mueller et al., 2010 study) and thus earlier cortico-cardiac correlation patterns. However, 
there are several similarities between the $\mathrm{N} 300 \mathrm{H}$ in the current study and $\mathrm{N} 300 \mathrm{H}$ in the Mueller et al study, such as latency in the EEG domain (300 ms), peak localization (centromedial electrodes) and polarity of the correlation (negative). Nevertheless, future studies are needed to clarify whether the effects of ATD reported here generalize to $\mathrm{N} 300 \mathrm{H}$ evoked by other tasks.

CECTs provide a method to measure cortico-cardiac coupling with higher temporal precision than neuroimaging-based approaches. However, when interpreting the latencies of $\mathrm{N} 300 \mathrm{H}$ in the present study, it should be considered, that IBI values only change with every heartbeat, and that an IBI value at a given time (e.g., at $250 \mathrm{~ms}$ ) is determined by the latency of the subsequent heart beat (which may occur several $100 \mathrm{~ms}$ later). Thus, the temporal resolution in the IBI domain is somewhat blurred. Nevertheless, under the assumption that $\mathrm{N} 300 \mathrm{H}$ is driven by the combined paths (a), (b), and (c), the present findings indicate that it took less than $1500 \mathrm{~ms}$ (i.e., $1750 \mathrm{~ms}$ minus $300 \mathrm{~ms}$ ) for the feedback signal to be transmitted from the cortex to the heart (or alternatively, to be simultaneously transmitted from a third region to the cortex and the heart). Thus, the cortical response $300 \mathrm{~ms}$ after a given feedback stimulus predicts how much the heart accelerates about one to two beats later.

As outlined in the introduction the $\mathrm{N} 300 \mathrm{H}$ and the P300 event-related potential show overlap with regard to latency and scalp topography. However, it should be emphasized that they are not the same phenomenon. The P300 reflects the averaged summed electrophysiological activity of several brain structures that become active around $300 \mathrm{~ms}$ after stimulus presentation (Soltani and Knight, 2000). The scalp P300, therefore, reflects a mixture (Makeig et al., 2002; Polich, 2007), which has previously been linked to a variety of phenomena including autonomous reactions, stimulus probability, motivational significance, attention, and task performance (Duncan-Johnson and Donchin, 1977; Isreal et al., 1980; Donchin et al., 1984; Li et al., 2009; Nieuwenhuis et al., 2011). In contrast, the N300H only reflects a highly specific portion of variance of EEG $300 \mathrm{~ms}$ after a feedback stimulus: that portion that is shared with variance of beat-to-beat intervals hundreds of ms later. Accordingly, N300H shows a distinct topography and latency (Mueller et al., 2010) and may be more differentially sensitive to manipulations of neurotransmitter systems than the overall P300 event-related potential.

In the present study, we were predominantly interested in the effect of 5-HT on cortico-cardiac connectivity in feedback processing. Following TRP- vs. TRP+, we found relatively lower $\mathrm{N} 300 \mathrm{H}$, which explains the previously reported finding in the same data, namely that ATD had no effect on cortical feedbackrelated signatures but attenuated the cardiac concomitants of feedback processing. These findings are consistent with a predominant role of 5-HT for path (c), which has been demonstrated in studies with cats and rats before (Jordan, 2005). However, given the widespread distribution of 5-HT in the human brain (Cools et al., 2008), future studies are necessary to further specify the particular path(s) that involves 5-HT for transmitting feedbackrelated information from the brain to the heart. Obviously, 5-HT may not be the only monoaminergic transmitter-system involved. Dopamine influences how errors (i.e., internal performance feedback) are processed at cortical sites such as the anterior (mid-) cingulate cortex and the effect of dopamine at these sites may be further modulated by 5-HT (Mueller et al., 2011). Moreover, widespread noradrenaline release triggered at the Locus Ceruleus following relevant stimuli such as feedback may lead to parallel modulations of P300-like amplitudes [i.e., upward arrow (a)] and visceral reactions [downward arrow (b)] and thereby induce or amplify a $\mathrm{N} 300 \mathrm{H}$ like covariation of $\mathrm{P} 300$ and cardiac acceleration (Nieuwenhuis et al., 2011). As a consequence we suggest that the monoaminergic mechanisms underlying $\mathrm{N} 300 \mathrm{H}$ are investigated in future studies by also using catecholaminergic challenge tests and/or molecular genetic assessments. In this regard, two particularly promising candidate genes are the widely studied COMT Val158Met polymorphism and a recently reported polymorphism in the promoter region of the norepinephrine transporter gene (Kim et al., 2006), both of which have previously been linked to P300 amplitude (Gallinat et al., 2003) and latency (Enge et al., 2011), respectively.

In the TRP + session, the tryptophan level predicted the amplitude of $\mathrm{N} 300 \mathrm{H}$. Individuals with higher plasma tryptophan levels, possibly associated with higher levels of 5-HT, showed a stronger covariation of cortical and cardiac activity after feedback stimuli. Accordingly, individual differences in cortico-cardiac connectivity may be biologically linked to 5-HT. Interestingly, both, 5-HT and cortico-cardiac connectivity may be of high relevance for individual differences in trait anxiety. For example, the short allele of the 5-HT transporter polymorphism (5-HTTLPR) predicts high levels of extracellular 5-HT and is associated with elevated risk for high trait anxiety and negative emotionality (Lesch et al., 1996; Sen et al., 2004), particularly in individuals who were previously exposed to adverse life events (Karg et al., 2011). Individuals who show stronger visceral reactions to cortically processed (feedback) information may also experience increased subjective anxiety more often, given that interoception of peripheral reactions (i.e., awareness of increased heart rate) may increase subjective anxiety (Paulus and Stein, 2006). Together, this raises the question, whether genetically driven differences in 5-HT activity may be linked to anxiety by modulating how potentially threatening information such as (negative) external feedback is transmitted from the brain to the viscera. Future studies examining serotoninergic polymorphisms, trait anxiety and $\mathrm{N} 300 \mathrm{H}$ like phenomena using large samples will be needed to address this hypothesis, which emerges from the present findings. Because gender is known to influence serotoninergic neurotransmission (Jovanovic et al., 2008), negative emotionality and vagal reactivity (Thayer et al., 1998), such studies should either focus on one gender (as in the present study) or-if sample sizes are sufficiently large-explicitly include gender as an independent variable.

It is worth noting, that the effects of ATD on evoked cardiac response are significant for the initial deceleratory (0-1250 ms) but not for the acceleratory phase, while the $\mathrm{N} 300 \mathrm{H}$, which is also affected by ATD, suggests an association between EEG and heart period from 250 to $1750 \mathrm{~ms}$. At the moment, detailed mechanistic explanations for this dissociation between evoked IBI and CECTs would be speculative. However, it should be noted, that cardiac responses are influenced by a variety of more or less opposing processes, which may occur in parallel. Accordingly, the 
observed cardiac response pattern (deceleration-accelerationdeceleration) may reflect the overall net effect of several (cortical and non-cortical) processes while the CECT only captures heart period fluctuations that covary with feedback-evoked cortical activity. The present $\mathrm{N} 300 \mathrm{H}$ findings indicate that increased (positive) EEG around $300 \mathrm{~ms}$ covaries with a relative cardiac acceleration from 250 to $1750 \mathrm{~ms}$ after TRP+ and that this covariation is lower or even reversed after TRP-. At which latency and to which degree this relative cardiac acceleration contributes to the net evoked IBI is a different question, which should be addressed in future studies.

Consistent with the assumption of parallel partially opposing brain-heart processes there appeared to be a positive correlation between feedback-evoked EEG and IBI in the TRP- session. Unlike the $\mathrm{N} 300 \mathrm{H}$, this association did not show a precise temporal or spatial localization, although the largest correlation cluster at $\mathrm{Cz}$ appeared from 300 to $400 \mathrm{~ms}$. One could speculate, that while earlier feedback-evoked centromedial brain activity (i.e., $200-300 \mathrm{~ms}$ ) is linked to cardiac acceleration, for example to prepare the organism for subsequent actions, more widespread brain activity with a later onset is linked to cardiac deceleration, possibly associated with information intake. If 5-HT differently contributes to these cortico-cardiac processes the polarity of the net CECT could be modulated by ATD as in the present study. However, it should be emphasized that this cortico-cardiac covariation in the TRP - session was unexpected with regard to polarity, spatial, and temporal localization. The current interpretations thus remain speculative until this pattern is replicated in a larger independent sample.

Two limitations deserve attention. First of all, the present study investigated a relatively small sample. Thus, although an $\mathrm{N} 300 \mathrm{H}$-like phenomenon was detected after TRP+, this component was not significantly different from zero after rigorously controlling for $n=500$ computed $t$-tests and applying a conservative significance level of $\alpha=0.0001$. To investigate the effect of TRP+ vs. TRP- however, the use of a cross-over design, allowed us to test substantially smaller samples than would be

\section{REFERENCES}

Bell, C., Abrams, J., and Nutt, D. (2001). Tryptophan depletion and its implications for psychiatry. Br. J. Psychiatry 178, 399-405.

Benarroch, E. E. (1997). Central Autonomic Network: Functional Organization and Clinical Correlations. Armonk, NY: Futura Pub. Co.

Berntson, G. G., Bigger, J. T. Jr., Eckberg, D. L., Grossman, P., Kaufmann, P. G., Malik, M., Nagaraja, H. N., Porges, S. W., Saul, J. P., Stone, P. H., and van der Molen, M. W. (1997). Heart rate variability: origins, methods, and interpretive caveats. Psychophysiology 34, 623-648.

Cools, R., Roberts, A. C., and Robbins, T. W. (2008). Serotoninergic regulation of emotional and behavioural

needed to achieve a comparable power with a between-subjects design. Moreover, the detected effects were relatively large and reached statistical significance at an uncorrected level $(p<0.005)$ despite the small sample. However, future studies using experimental manipulations of 5-HT with larger samples and other methods (e.g., using selective 5-HT reuptake inhibitors or molecular genetics) in combination with the CECT approach could further strengthen the present findings. As a second limitation, CECTs that were computed separately for positive and negative feedback stimuli may not have had enough trials for reliable within subject correlations. Because single-trial EEG and singletrial IBI show high levels of noise and non-specific fluctuations, respectively, individual CECT-correlations are typically relatively small $(r<0.2)$ and need a large number of trials to be reliable. Due to the small sample size and the relatively small number of feedback-specific trials, effects of feedback valence or interactions of feedback valence and ATD may not have had enough statistical power to reach significance in the present study. Although the lack of a main effect of feedback valence on $\mathrm{N} 300 \mathrm{H}$ is consistent with Mueller et al. (2010), interactions of feedback valence and 5-HT cannot be ruled out with the present findings. Particularly, the role of 5-HT in cortico-cardiac processing of negative feedback could be relevant in the abovementioned context of anxiety, which could be more closely investigated in future studies.

Despite these limitations, we showed for the first time, that the covariation of cortical and cardiac activity following external feedback presentation in humans is affected by plasma tryptophan availability, which determines the rate of central 5-HT synthesis. Our findings thereby provide indirect evidence that 5HT is of relevance for the interaction between the brain and the heart following feedback presentation.

\section{ACKNOWLEDGMENTS}

This study was supported by a TOP grant (912-02-050) from ZonMW-NWO to Dr. Evers and a DFG grant DFG WA 2593/2-2 to Dr. Wacker.

tional magnetic resonance imaging identifies the dynamics of performance monitoring. J. Neurosci. 25, 11730-11737.

Donchin, E. (1981). Presidential address, 1980. Surprise! ... Surprise? Psychophysiology 18, 493-513.

Donchin, E., Heffley, E., Hillyard, S. A., Loveless, N., Maltzman, I., Ohman, A., Rosler, F., Ruchkin, D., and Siddle, D. (1984). Cognition and event-related potentials. II. The orienting reflex and P300. Ann. N.Y. Acad. Sci. 425, 39-57.

Duncan-Johnson, C. C., and Donchin, E. (1977). On quantifying surprise: the variation of event-related potentials with subjective probability. Psychophysiology 14, 456-467.

Eichele, H., Juvodden, H. T., Ullsperger, M., and Eichele, T. (2010). Maladaptation of event-related EEG responses preceding performance errors. Front. Hum. Neurosci. 4:65. doi: 10.3389/fnhum.2010.00065

Enge, S., Fleischhauer, M., Lesch, K. P., Reif, A., and Strobel, A. (2011). Serotonergic modulation in executive functioning: linking genetic variations to working memory performance. Neuropsychologia 49, 3776-3785.

Fowles, D. C., Fisher, A. E., and Tranel, D. T. (1982). The heart beats to reward: the effect of monetary incentive on heart rate. Psychophysiology 19, 506-513.

Gallinat, J., Bajbouj, M., Sander, T., Schlattmann, P., Xu, K., Ferro, E. F., Goldman, D., and Winterer, G. (2003). Association of the G1947A COMT (Val(108/158)Met) gene polymorphism with prefrontal P300 during information 
processing. Biol. Psychiatry 54, 40-48.

Gianaros, P. J., van der Veen, F. M., and Jennings, J. R. (2004). Regional cerebral blood flow correlates with heart period and high-frequency heart period variability during working-memory tasks: implications for the cortical and subcortical regulation of cardiac autonomic activity. Psychophysiology 41, 521-530.

Graham, F. K., and Clifton, R. K. (1966). Heart-rate change as a component of the orienting response. Psychol. Bull. 65, 305-320.

Isreal, J. B., Chesney, G. L., Wickens, C. D., and Donchin, E. (1980). P300 and tracking difficulty: evidence for multiple resources in dual-task performance. Psychophysiology 17, 259-273.

Hajcak, G., McDonald, N., and Simons, R. F. (2003). To err is autonomic: error-related brain potentials, ANS activity, and post-error compensatory behavior. Psychophysiology 40, 895-903.

Holroyd, C. B., and Coles, M. G. (2002). The neural basis of human error processing: reinforcement learning, dopamine, and the errorrelated negativity. Psychol. Rev. 109, 679-709.

Jordan, D. (2005). Vagal control of the heart: central serotonergic (5-HT) mechanisms. Exp. Physiol. 90, 175-181.

Jovanovic, H., Lundberg, J., Karlsson, P., Cerin, A., Saijo, T., Varrone, A., Halldin, C., and Nordstrom, A. L. (2008). Sex differences in the serotonin $1 \mathrm{~A}$ receptor and serotonin transporter binding in the human brain measured by PET. Neuroimage 39, 1408-1419.

Karg, K., Burmeister, M., Shedden, K., and Sen, S. (2011). The serotonin transporter promoter variant (5-HTTLPR), stress, and depression meta-analysis revisited: evidence of genetic moderation. Arch. Gen. Psychiatry 68, 444-454.

Kim, C. H., Hahn, M. K., Joung, Y., Anderson, S. L., Steele, A. H., Mazei-Robinson, M. S., Gizer, I., Teicher, M. H., Cohen, B. M., Robertson, D., Waldman, I. D., Blakely, R. D., and Kim, K. S. (2006). A polymorphism in the norepinephrine transporter gene alters promoter activity and is associated with attentiondeficit hyperactivity disorder. Proc. Natl. Acad. Sci. U.S.A. 103, 19164-19169.

Lesch, K. P., Bengel, D., Heils, A., Sabol, S. Z., Greenberg, B. D., Petri, S., Benjamin, J., Muller, C. R., Hamer, D. H., and Murphy, D. L. (1996). Association of anxiety-related traits with a polymorphism in the serotonin transporter gene regulatory region. Science 274, 1527-1531.

Li, R., Keil, A., and Principe, J. C. (2009). Single-trial P300 estimation with a spatiotemporal filtering method. J. Neurosci. Methods 177, 488-496.

Linden, D. E. (2005). The P300: where in the brain is it produced and what does it tell us? Neuroscientist 11, 563-576.

Makeig, S., Westerfield, M., Jung, T. P., Enghoff, S., Townsend, J., Courchesne, E., and Sejnowski, T. J. (2002). Dynamic brain sources of visual evoked responses. Science 295, 690-694.

Manaker, S., and Verderame, H. M. (1990). Organization of serotonin $1 \mathrm{~A}$ and $1 \mathrm{~B}$ receptors in the nucleus of the solitary tract. J. Comp. Neurol. 301, 535-553.

Miltner, W. H. R., Braun, C. H., and Coles, M. G. H. (1997). Eventrelated brain potentials following incorrect feedback in a timeestimation task: evidence for a "generic" neural system for error detection. J. Cogn. Neurosci. 9, 788-798.

Mueller, E. M., Makeig, S., Stemmler, G., Hennig, J., and Wacker, J. (2011). Dopamine effects on human Error processing depend on catechol-O-methyltransferase VAL158MET genotype. J. Neurosci. 31, 15818-15825.

Mueller, E. M., Stemmler, G., and Wacker, J. (2010). Single-trial electroencephalogram predicts cardiac acceleration: a time-lagged P-correlation approach for studying neurovisceral connectivity. Neuroscience 166, 491-500.

Nieuwenhuis, S., De Geus, E. J., and Aston-Jones, G. (2011). The anatomical and functional relationship between the P3 and autonomic components of the orienting response. Psychophysiology 48, 162-175.

Notebaert, W., Houtman, F., Opstal, F. V., Gevers, W., Fias, W., and Verguts, T. (2009). Post-error slowing: an orienting account. Cognition 111, 275-279.

Panitz, C., Wacker, J., Stemmler, G., and Mueller, E. M. (2011) "Temporal coupling of cortical and cardiac activity," in Psychologie und Gehirn, eds D. Hagemann and P. Kirsch (Heidelberg: Universität Heidelberg), 199.

Paulus, M. P., and Stein, M. B. (2006). An insular view of anxiety. Biol. Psychiatry 60, 383-387.

Polich, J. (2007). Updating P300: an integrative theory of $\mathrm{P} 3 \mathrm{a}$ and $\mathrm{P} 3 \mathrm{~b}$. Clin. Neurophysiol. 118, 2128-2148.

Rushby, J. A., Barry, R. J., and Doherty, R. J. (2005). Separation of the components of the late positive complex in an ERP dishabituation paradigm. Clin. Neurophysiol. 116, 2363-2380.

Sato, A., Yasuda, A., Ohira, H., Miyawaki, K., Nishikawa, M., Kumano, H., and Kuboki, T. (2005). Effects of value and reward magnitude on feedback negativity and P300. Neuroreport 16, 407-411.

Sen, S., Burmeister, M., and Ghosh, D. (2004). Meta-analysis of the association between a serotonin transporter promoter polymorphism (5-HTTLPR) and anxiety-related personality traits. Am. J. Med. Genet. B Neuropsychiatr. Genet. 127B, 85-89.

Soltani, M., and Knight, R. T. (2000). Neural origins of the P300. Crit. Rev. Neurobiol. 14, 199-224.

Thayer, J. F., and Lane, R. D. (2007). The role of vagal function in the risk for cardiovascular disease and mortality. Biol. Psychol. 74, 224-242.

Thayer, J. F., Smith, M., Rossy, L. A., Sollers, J. J., and Friedman, B. H. (1998). Heart period variability and depressive symptoms: gender differences. Biol. Psychiatry 44, 304-306.

Thor, K. B., Blitz-Siebert, A., and Helke, C. J. (1992). Autoradiographic localization of 5HT1 binding sites in autonomic areas of the rat dorsomedial medulla oblongata. Synapse 10, 217-227.

Ullsperger, M. (2010). Genetic association studies of performance monitoring and learning from feedback: the role of dopamine and serotonin. Neurosci. Biobehav. Rev. 34, 649-659.

Ullsperger, M., and Von Cramon, D. Y. (2003). Error monitoring using external feedback: specific roles of the habenular complex, the reward system, and the cingulate motor area revealed by functional magnetic resonance imaging. J. Neurosci. 23, 4308-4314.

van der Veen, F. M., Mies, G. W., van der Molen, M. W., and Evers, E. A. (2008). Acute tryptophan depletion in healthy males attenuates phasic cardiac slowing but does not affect electro-cortical response to negative feedback. Psychopharmacology (Berl.) 199, 255-263.

Wager, T. D., Waugh, C. E., Lindquist, M., Noll, D. C., Fredrickson, B. L., and Taylor, S. F. (2009). Brain mediators of cardiovascular responses to social threat Part I: reciprocal dorsal and ventral sub-regions of the medial prefrontal cortex and heart-rate reactivity. Neuroimage 47, 821-835.

Wessel, J. R., Danielmeier, C., and Ullsperger, M. (2011). Error awareness revisited: accumulation of multimodal evidence from central and autonomic nervous systems. J. Cogn. Neurosci. 23, 3021-3036.

Conflict of Interest Statement: The authors declare that the research was conducted in the absence of any commercial or financial relationships that could be construed as a potential conflict of interest.

Received: 27 January 2012; accepted: 20 March 2012; published online: 09 April 2012.

Citation: Mueller EM, Evers EA, Wacker $J$ and van der Veen F (2012) Acute tryptophan depletion attenuates brainheart coupling following external feedback. Front. Hum. Neurosci. 6:77. doi 10.3389/fnhum.2012.00077

Copyright (C) 2012 Mueller, Evers, Wacker and van der Veen. This is an open-access article distributed under the terms of the Creative Commons Attribution Non Commercial License, which permits non-commercial use, distribution, and reproduction in other forums, provided the original author and source are credited. 